\title{
Adverse Reactions after Hepatitis B Vaccination: A Retrospective Analysis Using Spontaneous Reports
}

\author{
M. Kalaivani*1, Shruti Rastogi ${ }^{2}$, V Kalaiselvan ${ }^{3}$, G. N. Singh ${ }^{4}$ \\ 'Senior Scientific Officer, Biologics Section, Indian Pharmacopoeia Commission, Ministry of Health and Family Welfare (Govt. of India), Ghaziabad, INDIA. \\ 2Pharmacopoeial Associate, Biologics Section, Indian Pharmacopoeia Commission, Ministry of Health and Family Welfare (Govt. of India), Ghaziabad, INDIA. \\ ${ }_{3}^{3}$ Principal Scientific Officer, Indian Pharmacopoeia Commission, Ministry of Health and Family Welfare (Govt. of India), Ghaziabad, INDIA. \\ ${ }^{4}$ Secretary-cum-Scientific Director, Indian Pharmacopoeia Commission, Ministry of Health and Family Welfare (Govt. of India), Ghaziabad, INDIA.
}

\begin{abstract}
Background: Safety of vaccines is more critical because they are used for immunization in children and Hepatitis-B vaccination is included in national immunization schedule for neonates and infants in India. The National Coordination Centre (NCC)-Pharmacovigilance Programme of India (PvPI), Indian Pharmacopoeia Commission (IPC) monitors the adverse drug reactions/Adverse events (ADR/AE) associated with the drugs used in the country through WHO reporting system, VigiFlow. Objective of the study is to analyse ADR/AEs associated with Hepatitis B vaccination reported to NCC-PvPI through VigiFlow database. Methods: Retrospective analysis of the Vigiflow data were extracted and analysed with respect to patient demography, profession of reporter, system organ class (SOC) affected, causality assessment, seriousness of adverse event and year wise analysis of reported ADRs. Results: During the study period 295 ADR/AEs were reported following Hepatitis B vaccination. The adverse reactions following Hepatitis B immunization were primarily reported in infants $(55.2 \%)$ in age group $1-2$ years. The causality assessment showed that $42.7 \%$ of the adverse events are probable/likely associated with Hepatitis B vaccination. Most of the cases
\end{abstract}

were reported by the physician. The two main ADRs were fever $58.9 \%$ and injection site pain $11.5 \%$. Conclusion: Data analyzed in our study showed only non-serious reactions were reported due to hepatitis $B$ vaccination. Data also clearly showed that there is a need for reporting ADR/AEs associated with quality of vaccines that may affect safety of the vaccine, which may be possible by amending the suspected adverse drug reaction reporting form of NCC-PvPI.

Key words: NCC-PvPI; Hepatitis B vaccine; ADR/AE; VigiFlow; system organ class (SOC)

Correspondence :

*Dr. M. Kalaivani,

Senior Scientific Officer, Biologics Section, Indian Pharmacopoeia Commission, Ministry of Health and Family Welfare, Govt. of India,Sector-23, Raj Nagar, Ghaziabad-201 002, INDIA

E-mail: kala_samy2002@yahoo.com

DOI: 10.5530/jyp.2017.9.11

\section{INTRODUCTION}

More than 780 thousand people die every year due to complications of Hepatitis B such as chronic hepatitis to active hepatitis, cirrhosis, hepatocellular carcinoma etc caused by Hepatitis B Virus (HBV). ${ }^{1}$ They are transmitted from one person to other person through blood or other body fluids or from mother to child at the time of birth. Perinatal transmission of HBV leads to more than one third of hepatitis B carriers. ${ }^{2}$ Chronic hepatitis B is more prevalent at a younger age and approximately $90 \%$ of infected infants develop chronic infections.

To prevent $\mathrm{HBV}$ infection, vaccine against hepatitis $\mathrm{B}$ was available since 1982, which is a hepatitis B surface antigen (HBsAg) purified from the plasma of HBV infected persons, with the evolution of science recombinant derived hepatitis B vaccine was developed in the year 1997 in India. ${ }^{3}$ The only difference between plasma-derived and recombinant hepatitis B particle is glycosylation of proteins. ${ }^{3}$ Ping-Ing Lee and Chin-Yun Lee in the year 1998 reported that the safety, immunogenicity and protective efficacy of recombinant derived Hepatitis B Vaccine is similar to that of plasma-derived. ${ }^{4}$ In India, hepatitis B vaccine is available as single vaccine formulations or in combination with other vaccines such as diphtheria-tetanus-pertussis (DTP), Haemophilus influenza type b, hepatitis $\mathrm{A}$, inactivated polio and recombinant hepatitis $\mathrm{B}$ vaccines. ${ }^{5}$ Hepatitis $B$ vaccine is usually given as 3-4 dosed over a 6 month period upto 18 months of age. ${ }^{6}$
Vaccines are subjected to extensive premarket safety testing before their approval, but certain adverse effects are not identified in preclinical and clinical phase. In India safety of vaccines is monitored by the Immunization Division under Ministry of Health and Family Welfare, Government of India through Adverse Event Following Immunization (AEFI) division and Pharmacovigilance Programme of India (PvPI) and the regulatory interventions on vaccines taken by Central Drugs Standard Control Organisation (CDSCO), a national regulatory authority. ${ }^{7,8}$ AEFI division prescribed a guideline for reporting $\mathrm{AE}$ after immunization, irrespective of dose numbers. AEFI division also prescribed format for submitting the First Information Report (FIR), Preliminary Investigation Report (PIR) and Detailed Investigation Report (DIR) by periphery, district and state health authorities for collating adverse event after immunization and will help in its causality assessment. The HCPs should submit FIR to district immunization officer (DIO) for reporting serious AEFI cases and district AEFI committee members will then investigate the reports further, ${ }^{8}$ after investigation DIO should submit the reports of the same to state and national level offices. ${ }^{8}$ Causality assessment is the systematic review AEFI case; it aims to determine the likelihood of a causal association between the event and the vaccine(s) received. Causality assessments were done by state AEFI committee based on its guideline ${ }^{8}$ and WHO guideline for 'Causality assessment of adverse event following immunization' ${ }^{10}$ at state level or national level.

To ensure the safety of drugs and pharmaceuticals in the country including vaccines, the Indian Pharmacopoeia Commission (IPC) is 
acting as the National Coordination Centre (NCC) for PvPI starting on April 15, 2011. ${ }^{9}$ The NCC works with the responsibility of monitoring all the adverse reactions of the medicines used in India and to develop and maintain Pharmacovigilance database for patient safety. NCC also participates in the WHO international Drug Monitoring Programme and collaborating with WHO-Uppsala Monitoring Centre (UMC), Sweden. The HCPs can report AEFI cases to ADR monitoring centers (AMC) or NCC-PvPI, and then PvPI coordinates with national level AEFI committee and State Expanded Programme Immunization Officers for reporting and investigation. Further AEFI committee follows with state and district team to complete the FIR/PIR/DIR to do causality assessment. ${ }^{9}$ To NCC-PvPI AEFIs were reported through VigiFlow, spontaneous online reporting system developed by the UMC for international drug monitoring and/or by submitting 'Suspected Adverse Drug Reaction Reporting Form' which is available on the official website of IPC (www.ipc.gov.in). ${ }^{9}$

The aim of this study was to review and analyze adverse events (AEs) and causality assessment associated with hepatitis $\mathrm{B}$ vaccine reported to NCC-PvPI with a focus on comparison of the safety data associated with plasma-derived hepatitis $B$ vaccine with recombinant hepatitis $B$ vaccine and to analyze reports associated with concomitant administration of other vaccines.

\section{METHODS}

A retrospective analysis of Individual Case Safety Reports (ICSRs) related to plasma-derived and recombinant hepatitis B vaccine reported to PvPI through spontaneous online reporting system VigiFlow, developed by the Uppsala Monitoring Centre (UMC), the World Health Organization (WHO)'s collaborating center for international drug monitoring. All ADR/AEs reported to NCC-PvPI, IPC, Ghaziabad, India till $31^{\text {st }}$ March 2016 were studied. The NCC has been associated with 150 teaching / multispecialty hospitals as ADR Monitoring Centers (AMCs) throughout India to monitor the safety of drugs in the country. The data were collected from the reports with/without concomitant drugs and with reaction level preferred. The data were analyzed with respect to the patient demography, profession of reporter, causality assessment, year wise analysis of reported primary SOC as per the MedDRA, age and gender of the patients, the severity of the report and the relatedness of the product using 'Search and statistics' tool in VigiFlow. ${ }^{11}$

\section{RESULTS}

Data from India reported to VigiFlow from $23^{\text {rd }}$ December 2010 to $31^{\text {st }}$ March 2016 were analyzed. Though IPC started functioning as PvPI from 2011, AMCs had reported previous data of December 2010 also. Out of 2107 ICSRs related to vaccines submitted to NCC-PvPI, 295 ICSR's were related to Hepatitis B vaccines, which contributes $14 \%$ of ADR/ AEs related to vaccines. The data were analysed for patient demography, profession of reporter, causality assessment, year wise analysis of reported primary SOC as per the MedDRA, age and gender of the patients, the severity of the report and the relatedness of the product were analysed as per WHO-UMC classification.

Patient demography of data showed that $44 \%(n=130)$ ADR/AEs were reported from male, $44.4 \%(n=131)$ were from females, whereas $11.5 \%$ $(\mathrm{n}=34) \mathrm{ADR} / \mathrm{AEs}$ were not classified as gender wise classification . It was also observed that $61.0 \%(n=180) A D R / A E s$ were reported from the age group of $1-4$ years, $21.3 \%(n=63)$ of the cases were reported from neonates and $1 \%(n=3)$ cases were reported from adult age group of 44-46 yrs (Table 1). One report was reported from a 46 year old woman immunized with Hepatitis B vaccine was reported with difficulty in breathing and bronchospasm, a serious reaction appeared immediately after immunization with one dose. The reaction was managed by with-
Table 1: A data analysis on Patient demography and Profession of reporter from the ICSRs after Hepatitis-B vaccination reported to NCC-PvPI during the study period

\begin{tabular}{|c|c|c|}
\hline S. No. & Characteristic & No. of cases of $(n=295)[n(\%)]$ \\
\hline \multirow[t]{4}{*}{1} & Gender & \\
\hline & Male & $130(44.0)$ \\
\hline & Female & $131(44.4)$ \\
\hline & Unknown & $34(11.5)$ \\
\hline \multirow[t]{12}{*}{2} & Age Group & \\
\hline & Neonates (0-30 days) & $63(21.3)$ \\
\hline & Infants & \\
\hline & - $1-<2$ years & $163(55.2)$ \\
\hline & Children & \\
\hline & - $2-<3$ years & $10(3.3)$ \\
\hline & - $3-<5$ years & $7(2.3)$ \\
\hline & - $\geq 5$ years & $5(1.7)$ \\
\hline & Adults & \\
\hline & - $18-44 \mathrm{yr}$ & $15(5.0)$ \\
\hline & - (44-46yr) & $3(1.0)$ \\
\hline & Unknown & $29(9.8)$ \\
\hline \multirow[t]{4}{*}{3} & Profession of reporter & \\
\hline & Physician & $246(83.3)$ \\
\hline & Pharmacist & $3(1.0)$ \\
\hline & Other healthcare professional & $46(15.5)$ \\
\hline
\end{tabular}

${ }^{*}$ ICSR-Individual Case safety reports; NCC- National Coordination Centre; PvPIPharmacovigilance Programme of India

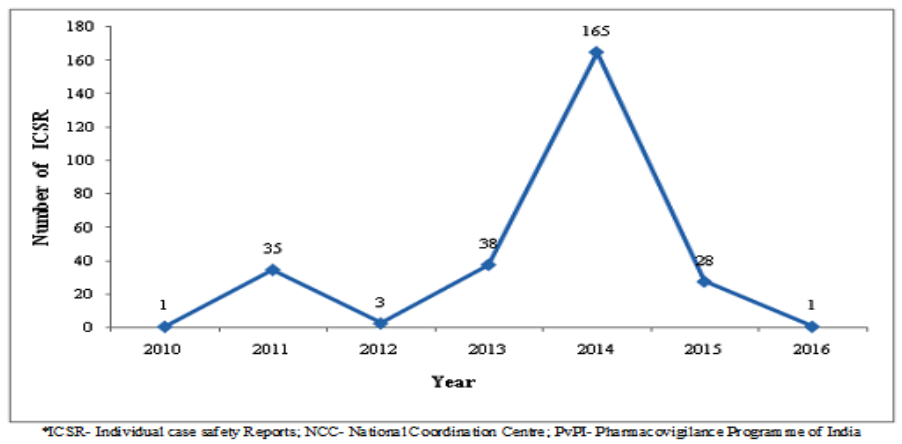

Figure 1: Year wise analysis of ICSR submitted to NCC-PvPI.

drawing the medication, as reported by pharmacist (3\%) in the report. Except this all other ADR/Es were reported by physicians (83.3\%) (Table 1). $\mathrm{ADR} / \mathrm{AEs}$ due to hepatitis B vaccination reported as per SOC of MedDRA is depicted in Table 2. VigiFlow data showed 295 cases from December 2010 including the concomitant drug reports. When classified by system organ, the most common ADR/AEs reported were 'body as a whole- general' disorders $(n=197 ; 66.7 \%)$ including death $(n=1)$, oedema $(n=2)$, fever $(n=174)$, pain $(n=4)$, limb pain $(n=1)$ and crying abnormal $(n=15)$; Skin and appendages $(n=8 ; 2.7 \%)$ including eczema $(n=1)$, pruritis $(n=1)$, rash $(n=4)$ and urticaria $(n=2)$; Musculo-skeletal system including myalgia $(n=3 ; 1 \%)$; neurological disorder $(n=7 ; 2.3 \%)$ including coma $(\mathrm{n}=1)$, convulsions $(\mathrm{n}=2)$, complex partial seizures $(\mathrm{n}=1)$ and febrile convulsions $(n=3)$; Psychiatric disorders $(n=1 ; 0.3 \%)$; Gastro-intestinal disorders $(n=10 ; 3.3 \%)$, including diarrhoea $(n=8)$ and vomiting $(n=2)$; Cardiovascular disorders $(n=3 ; 1 \%)$, including cardiac arrest $(n=1)$ and cyanosis $(n=2)$; Respiratory system disorder $(n=7 ; 2.3 \%)$, including bronchospasm $(n=1)$, coughing $(n=1)$, dyspnoea $(n=2)$, pharyngitis 
Table 2: A data analysis on SOC of ADR reported to NCC-PvPI following Hepatitis B vaccination during the study period

\begin{tabular}{|c|c|c|c|c|}
\hline \multirow{2}{*}{ SOC } & \multirow{2}{*}{ SOC disorders } & \multicolumn{2}{|l|}{ ADR reported } & \multirow{2}{*}{$\begin{array}{l}\text { Hepatitis B Vaccine } \\
\qquad(n=295)[n(\%)]\end{array}$} \\
\hline & & Reaction & $\mathrm{n}$ & \\
\hline \multirow{4}{*}{0100} & \multirow{4}{*}{ Skin and appendages } & Eczema & 1 & \multirow{4}{*}{$8(2.7)$} \\
\hline & & Pruritis & 1 & \\
\hline & & Rash & 4 & \\
\hline & & Urticaria & 2 & \\
\hline 0200 & Musculo-skeletal system & Myalgia & 3 & $3(1.0)$ \\
\hline \multirow{4}{*}{0400} & \multirow{4}{*}{ Neurological disorders } & Coma & 1 & \multirow{4}{*}{$7(2.3)$} \\
\hline & & Convulsions & 2 & \\
\hline & & Complex partial seizures & 1 & \\
\hline & & Febrile convulsions & 3 & \\
\hline 0500 & Psychiatric disorders & Somnolence & 1 & $1(0.3)$ \\
\hline \multirow{2}{*}{0600} & \multirow{2}{*}{ Gastro-intestinal system } & Diarrhoea & 8 & \multirow{2}{*}{$10(3.3)$} \\
\hline & & Vomiting & 2 & \\
\hline \multirow{2}{*}{1000} & \multirow{2}{*}{ Cardiovascular disorder } & Cardiac arrest & 1 & \multirow{2}{*}{$3(1.0)$} \\
\hline & & Cyanosis & 2 & \\
\hline \multirow{5}{*}{1100} & \multirow{5}{*}{ Respiratory system disorders } & Bronchospasm & 1 & \multirow{5}{*}{$7(2.3)$} \\
\hline & & Coughing & 1 & \\
\hline & & Dyspnoea & 2 & \\
\hline & & Pharyngitis & 2 & \\
\hline & & Respiratory arrest & 1 & \\
\hline \multirow{3}{*}{1600} & \multirow{3}{*}{ Neonatal and infancy disorders } & Convulsions neonatal & 1 & \multirow{2}{*}{$5(1.7)$} \\
\hline & & Fever neonatal & 4 & \\
\hline & & Oedema generalized & 2 & \multirow{6}{*}{$197(66.7)$} \\
\hline \multirow{5}{*}{1810} & \multirow{5}{*}{ Body as a whole - general disorders } & Death & 1 & \\
\hline & & Fever & 174 & \\
\hline & & Pain & 4 & \\
\hline & & Limb pain & 1 & \\
\hline & & Crying abnormal & 15 & \\
\hline \multirow{8}{*}{1820} & \multirow{8}{*}{ Application site disorders } & Application site oedema & 1 & \\
\hline & & Injection site mass & 1 & \\
\hline & & Injection site pain & 34 & \\
\hline & & Injection site reaction & 11 & $52(176)$ \\
\hline & & Injection site rash & 2 & \\
\hline & & Injection site bleeding & 1 & \\
\hline & & Injection site inflammation & 1 & \\
\hline & & Injection site infection & 1 & \\
\hline 1020 & 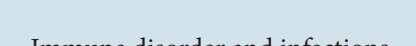 & Abscess & 1 & 20 \\
\hline 1000 & 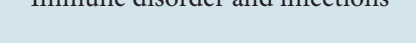 & Serum sickness & 1 & \\
\hline
\end{tabular}

*SOC-System organ class; ADR- Adverse drug reaction; NCC- National Coordination Centre; PvPI- Pharmacovigilance Programme of India 
Kalaivani et al.: Pharmacovigilance of Hepatitis B vaccine

Table 3: A data analysis on Causality assessment of ADR reported to NCC-PvPI following Hepatitis B vaccination during the study period

\begin{tabular}{|c|c|c|c|c|c|c|c|c|}
\hline \multirow[b]{2}{*}{ S.No } & \multirow[b]{2}{*}{$\begin{array}{l}\text { Hepatitis B vaccine } \\
\qquad(\mathrm{n} ; \%)\end{array}$} & \multicolumn{7}{|c|}{ Causality assessment ( $n=295)$} \\
\hline & & $\begin{array}{l}\text { Very likely } \\
\text { [n(\%)] }\end{array}$ & $\begin{array}{c}\text { Probable/ } \\
\text { Likely } \\
{[n(\%)]}\end{array}$ & $\begin{array}{c}\text { Possible } \\
\text { [n(\%)] }\end{array}$ & $\begin{array}{l}\text { Certain } \\
\text { [n(\%)] }\end{array}$ & $\begin{array}{l}\text { Unlikely } \\
\text { [n(\%)] }\end{array}$ & $\begin{array}{l}\text { Fatal } \\
{[n(\%)]}\end{array}$ & $\begin{array}{c}\text { Not clear } \\
{[n(\%)]}\end{array}$ \\
\hline \multirow{3}{*}{1.} & Recombinant Hepatitis B vaccine $(\mathrm{n}=90 ; 30.5)$ & 0 & $25(27.7)$ & $11(12.2)$ & $24(26.6)$ & $3(3.3)$ & 0 & $27(30)$ \\
\hline & Plasma derived Hepatitis B vaccine $(n=28 ; 9.4)$ & 0 & $22(78.5)$ & $4(14.2)$ & 0 & 0 & 0 & $2(7.1)$ \\
\hline & Hepatitis B vaccine\# $(\mathrm{n}=177 ; 60)$ & 0 & $79(44.6)$ & $24(13.5)$ & $43(24.2)$ & 0 & 0 & $31(17.5)$ \\
\hline \multirow{2}{*}{2.} & $\begin{array}{l}\text { Single vaccine } \\
(\mathrm{n}=34 ; 11.5)^{*}\end{array}$ & 0 & $18(52.9)$ & $11(32.3)$ & $3(8.8)$ & 0 & 0 & $2(5.8)$ \\
\hline & $\begin{array}{l}\text { In Combination vaccine } \$ \\
\qquad(\mathrm{n}=261 ; 88.4)\end{array}$ & 0 & $107(40.9)$ & $37(14.1)$ & $65(24.9)$ & $3(1.1)$ & 0 & $49(18.7)$ \\
\hline
\end{tabular}

${ }^{\star}$ Single vaccine- Hepatitis B vaccine (recombinant or plasma derived) as a individual vaccine and not in combination with any other vaccine;\#Hepatitis B vaccineMay be recombinant derived or plasma derived hepatitis B vaccine, not clear from the ICSR; $\$$ In Combination vaccine-Hepatitis B vaccine in combination with other vaccines such as DTP vaccines or Haemophilus type B vaccine or Hepatitis A vaccine

$(\mathrm{n}=2)$ and respiratory arrest $(\mathrm{n}=1)$; neonatal and infancy disorders $(\mathrm{n}=5$; $1.7 \%)$ including convulsions neonatal $(\mathrm{n}=1)$ and fever neonatal $(\mathrm{n}=4)$; application site disorders $(\mathrm{n}=52 ; 17.6 \%)$ including application site oedema $(n=1)$, injection site mass $(n=1)$, injection site pain $(n=34)$, injection site reaction $(n=11)$, injection site rash $(n=2)$, injection site bleeding $(\mathrm{n}=1)$ and injection site infection $(\mathrm{n}=1)$; Immune disorder and infections $(n=2 ; 0.6 \%)$ including one report each of abscess and serum sickness.

As mentioned in Table 3, the causality assessment of Hepatitis B vaccine showed that $30.5 \%(n=90)$ ICSRs are of recombinant Hepatitis B vaccine, $9.4 \%(n=28)$ of plasma-derived vaccine and in $60 \%(n=177)$ ICSRs the nature/method of production of hepatitis $\mathrm{B}$ vaccine was not mentioned. Among the reported ADR/AEs $27.7 \%$ are determined to be 'probable/likely' causally associated with recombinant hepatitis B vaccine and 78.5\% plasma-derived hepatitis B vaccine. In total $42.7 \%$ of the adverse events are probable/likely associated with Hepatitis B vaccination, irrespective of method of production. Table 3 also showed that ADR/AEs due to Hepatitis $B$ vaccine in combination with other vaccines such as DTP vaccine or heamophilus type $B$ vaccine or hepatitis $A$ vaccine is more $(\mathrm{n}=261 ; 88.4 \%)$ as compared to that of hepatitis $\mathrm{B}$ vaccine as andividual vaccine $(\mathrm{n}=34 ; 11.5 \%)$.

Figure 1 explains ADR/AEs reported year wise, maximum number $(\mathrm{n}=171 ; 61.5 \%)$ of ADR/AEs were reported in the year 2014, . Anarithmetic increase was observed in number of ADR/AEs since year 2010, which may be due to improved culture of ADR/AEs reporting to NCC in India among healthcare professionals.

\section{DISCUSSION}

Recombinant hepatitis B vaccine is the first recombinant product in the market, after its introduction in the year 1997 recombinant market is growing significantly and safety issues related to recombinant biologics also increased proportionately. ${ }^{12}$ Biologics including recombinant vaccines are heterogeneous and may contain aggregates, oxidized and deamidated molecules that could affect the safety and efficacy of the product. Vaccines are considered to be safe and effective for many decades and are successful in eradicating many infectious diseases such as polio caused by wild polioviruses ${ }^{13}$ small pox etc but the risk-benefit can be effectively analyzed only by using effective post-marketing pharmacovigilance system in the country. Hepatitis B vaccine is effective in prevention of acute and chronic hepatitis B infection. ${ }^{14,15}$ Immunization with Hepatitis B vaccine is recommended by WHO, National
Immunization Programme of the country and other international organizations for newborns, infants, adolescents and high risk adults. ${ }^{16}$

In India, National Immunization Schedule recommends hepatitis B vaccine in three doses at zero, 3 weeks and 6 months of age and if not vaccinated at the recommended age, children and adolescents as well as subjects who are, or will be, at increased risk of infection can be vaccinated at recommended intervals. ${ }^{17}$ The study showed that maximum number of ICSRs (82.3\%) were reported from the age group of 1-4 years, which may support that infant vaccination is good practice as compared to that of vaccination in children. WHO reports states that Hepatitis B vaccine is one of the safest vaccines but may induce some mild reactions includes soreness and fever. As per WHO, 15\% of adults and 5\% of children have tenderness, redness or mild swelling at injection site and about 1-6\% may develop a mild fever that lasts for one or two days after injection of the vaccine. ${ }^{18}$ Our study also confirmed the same that the two main adverse drug reactions reported were fever $58.9 \%(n=174)$ and injection site pain $11.5 \%(\mathrm{n}=34)$.

Proportionate increase in number of reports submitted to PvPI, clearly suggests that there is a proportionate increase in awareness and reporting culture among healthcare professionals. Among 295 ICSRs, 60\% $(\mathrm{n}=177)$ AEs are not classified as Plasma derived or recombinant derived Hepatitis B vaccine, though the details related to the production method/nature of origin is clearly mentioned in label of the vaccines as per the requirement of part X of Drugs and Cosmetics act 1940 and rules thereunder. ${ }^{19}$ Moreover 'Suspected Adverse Drug Reaction Reporting Form' of NCC-PvPI also demands the details on patient information, suspected adverse reaction, suspected medication and reporter information but not the information on manufacturing method. ${ }^{9}$

The present study shows that $22.7 \%$ of hepatitis vaccine ICSRs were 'certain' in causality assessment. In case of vaccines, to grade them as 'certain' detailed investigation report is required. In case of spontaneous reporting establishing 'certain' relation between vaccine and $\mathrm{AE}$ is the challenging part for HCPs. ${ }^{20}$ Therefore, to ensure the causality assessment reports were further communicated to AEFI division. Also, we cannot correlate these adverse events due to safety of vaccine; it may also due to the administrative or programmatic error.

Reporting rate was rapidly increased in the year 2014, because NCC organized several skill development programmes such as conferences, CME's etc to create awareness among healthcare providers and public on reporting of ADR's. Also NCC provided helpline number facility for reporting ADR's. 
Shinefield et al in the year 2006 and Hesley et al in the year 2004 reported that concomitant administration of MMRV, Hib/HepB and DTaP and bivalent Haemophilus influenzae type b-hepatitis $\mathrm{B}$ vaccine, measlesmumps-rubella vaccine and varicella vaccine respectively are well-tolerated and are safe and immunogenic. Though the authors don't have the data on proportion of population received combination vaccine rather than single vaccine but the analysis of data from PvPI revealed that the AEs associated with concomitant administration of vaccines are more than individual hepatitis B vaccine. ${ }^{21,22}$ This warrants for elaborate postmarketing collection of ADR/AEs due to hepatitis B vaccine.

According to Schedule Y of the Drugs and Cosmetics Act 1940, postmarketing surveillance should be conducted and the Periodic Safety Updates Reports (PSURs) should be submitted to regulatory authority every six months initially for a period of two years and then annually for another two years, serious and unexpected AEs should be reported within 15 days of its report. ${ }^{18}$ Apart from spontaneous reporting, postmarketing pharmacovigilance plan for the vaccines should also include submission of PSURs to NCC-PvPI and targeted spontaneous reporting of AEs due to these vaccines can also be promoted to ensure safety of vaccines in the country.

\section{CONCLUSION}

Our study clearly justifies the need for amending the AEs reporting form of NCC-PvPI (especially for biologics) to include production method, proprietary name (brand) name, INN, batch/lot number, manufacturer's name, country of origin, lack of efficacy, less effective and over effective, ${ }^{23,24}$ which leads to effective monitoring of safety of vaccines in the country and this may also give informations on efficacy of vaccines, additionally.

Most of the vaccines are administered in the pediatrics and special conditions, pharmacovigilance of vaccines is of prime importance for the country and the AEFI at central and state level are doing the same but there is a need for awareness among the healthcare professionals and patients to report the ADR/AEs associated with quality of vaccines that affect their safety and efficacy and also to indicate the nature of origin in the report while reporting the AEs to NCC-PvPI.

Ethical Approvals: Ethical approval was not required.

Funding: No sources of funding were used to assist in the preparation of this study.

\section{ACKNOWLEDGEMENT}

Authors acknowledges NCC-PvPI for providing technical support.

\section{CONFLICT OF INTEREST}

M. Kalaivani, Shruti Rastogi, V Kalaiselvan, G. N. Singh have no conflicts of interest that are directly relevant to the content of this study.

\section{ABBREVIATIONS USED}

ADR/AE: Adverse drug reaction/Adverse event; AEFI: Adverse Event Following Immunization; CDSCO: Central Drugs Standard Control Organisation; DIO: District immunization officer (DIO); DIR: Detailed Investigation Report; DTP: Diphtheria-tetanus-pertussis; FIR: First Information Report; HBsAg: Hepatitis B surface antigen; HBV: Hepatitis B Virus; ICSRs: Individual Case Safety Reports; IPC: Indian Pharmacopoeia Commission; MedDRA: Medical Dictionary for Regulatory Activities; NCC: National Coordination Centre; PIR: Preliminary Inves- tigation Report; PSUR: Periodic Safety Updates Reports; PvPI: Pharmacovigilance Programme of India; SOC: System organ class; UMC: Uppsala Monitoring Centre; WHO: World Health Organization.

\section{REFERENCES}

1. World Health Organization. Hepatitis B Fact Sheet. 2014. Geneva (CH) http:// www.who.int/mediacentre/factsheets/fs204/en/. Accessed 6 Nov 2014.

2. Yao JL. Perinatal transmission of hepatitis $B$ virus infection and vaccination in China. Gut. 1996; 38 Suppl 2: S37-S8

3. Weekly epidemiological record. 2009. http://www.who.int/wer/2009/wer 8440 pdf. Accessed 6 Nov 2014

4. Lee PI, Lee CY. Practical Considerations in Converting from Plasma-Derived to Recombinant Hepatitis B Vaccines. Bio Drugs. 1998;10:11-25.

5. Indian Pharmacopoeia Commission. Indian Pharmacopoeia. Ghaziabad, India: Ministry of Health and Family Welfare; 2014

6. Hepatitis B. Available from http://www.vaccines.gov/diseases/hepatitis_b/. Accessed 15 April 2015

7. Guidelines on Similar Biologics: Regulatory Requirements for Marketing Authorization in India. 2012. Central Drugs Standard Control Organisation.New Delhi (IN). http://www.cdsco.nic.in/writereaddata/Bio\%20Similar\%20 Guideline.pdf . Accessed 12 Nov 2014.

8. Government of India. Adverse Events Following Immunization: Surveillance and Response Operational Guidelines. New Delhi: Ministry of Health and Family Welfare, Government of India; 2010

9. Pharmacovigilance Programme of India (PvPI) for Assuring Drug Safety. Indian Pharmacopoeia Commission. Ghaziabad (IN). http://www.ipc.gov.in/ PvPl/pv_home.html. Accessed 12 Nov 2014

10. Causality assessment of adverse event following immunization (AEFI): use manual for the revised WHO classification. WHO/HIS/EMP/OSS. WHO Press, World Health Organization, 20 Avenue Appia, 1211 Geneva 27, Switzerland; 2013.

11. VigiFlow ${ }^{\mathrm{TM}}$. Available from http://www.whoumc.org/DynPage.aspx?id=97223 $\& m n 1=7347 \& m n 2=7252 \& m n 3=7254$. Accessed 14 Nov 2014

12. Bryan $A L$, Timothy $M$. Emerging patient safety issues under health care reform: follow-on biologics and immunogenicity. Ther Clin Risk Manag. 2011;7:489-93.

13. Jacob JT, Vipin MV. Eradicating poliomyelitis: India's journey from hyperendemic to polio-free status. Indian J Med Res. 2013;137(5):881-94

14. Margolis HS. Prevention of acute and chronic liver disease through immunization: hepatitis B and beyond. J Infect Dis. 1993;168:9-14

15. Lemon SM, Thomas DL. Vaccines to prevent viral hepatitis. N Engl J Med 1997;336:196-204.

16. Velu V, Nandakuamr S, Shanmugam S, Jadhav SS, Kulkarni PS, Thyagarajan SP. Comparison of three different recombinant hepatitis B vaccines: GeneVac-B, Engerix B and Shanvac B in high risk infants born to HBsAg positive mothers in India. World J Gastroenterol. 2007;13:3084-89.

17. Vipin MV, Panna C, Ajay K, Anuradha B, Naveen T, Vijay NY, et al. Indian Academy of Pediatrics (IAP) Recommended Immunization Schedule for Children Aged 0 through 18 years-India, 2014 and Updates on Immunization. Indian Pediatrics. 2014;51:785-800. http://www.indianpediatrics.net/oct2014/785.pdf

18. Hepatitis B-The vaccine. 2011. http://www.who.int/immunization/topics/ hepatitis_b/en/index2.html. Accessed 16 Nov 2014.

19. Malik V. Law relating to Drugs and Cosmetics. 22 edition 2011 and with supplement 2012. Eastern Book Company, Lucknow.

20. Palleria C, Leporini C, Chimirri S, Marrazzo G, Sacchetta S, Bruno L, et al. Limitations and obstacles of the spontaneous adverse drugs reactions reporting: Two "challenging" case reports. J Pharmacol Pharmacother. 2013;4(Suppl1):S66S72.

21. Shinefield H, Black S, Thear M, Coury D, Reisinger K, Rothstein E, et al. Safety and immunogenicity of a measles, mumps, rubella and varicella vaccine given with combined Haemophilus influenzae type $b$ conjugate/hepatitis $B$ vaccines and combined diphtheria-tetanus-acellular pertussis vaccines. Pediatr Infect Dis J. 2006;25(4):287-92.

22. Hesley TM, Reisinger KS, Sullivan BJ, Jensen EH, Stasiorowski S, Meechan C, et al. Concomitant administration of a bivalent Haemophilus influenzae type b-hepatitis $B$ vaccine, measles-mumps-rubella vaccine and varicella vaccine: safety, tolerability and immunogenicity. Pediatr Infect Dis J. 2004;23(3):240-5.

23. Shanthi N Pal, Chris D, Dennis F, Sten O. WHO Strategy for Collecting Safety Data in Public Health Programmes: Complementing Spontaneous Reporting Systems. Drug Saf. 2013;36(2):75-81.

24. WHO Expert Committee on Biological Standardization. Sixtieth report. WHO Technical Report Series 977. WHO press, WHO, Geneva, Switzerland. 